\title{
Relevance of Incorporating Cavity Shape Change in Modelling the Ductile Failure of Metals
}

\author{
Mohand Ould Ouali \\ Laboratory Elaboration and Characterization of Materials and Modelling (LEC2M), University Mouloud MAMMERI of Tizi Ouzou, \\ BP 17RP, 15000 Tizi Ouzou, Algeria
}

Correspondence should be addressed to Mohand Ould Ouali; m_ouldouali@ummto.dz

Received 28 February 2018; Revised 29 July 2018; Accepted 28 August 2018; Published 16 October 2018

Academic Editor: Stefano de Miranda

Copyright (C) 2018 Mohand Ould Ouali. This is an open access article distributed under the Creative Commons Attribution License, which permits unrestricted use, distribution, and reproduction in any medium, provided the original work is properly cited.

\begin{abstract}
The purpose of this paper is to assess the relevance of considering the cavities shape change in the context of physically based modelling of the ductile rupture in metals. Two thermomechanical models have been used in this study: the GTN model, developed by Gurson, Tvergaard, and Needleman for spherical cavities keeping their shape unchanged during loading, and the GLD model, proposed by Gologanu, Leblond, and Devaux for ellipsoidal cavities that can change their shape. The GTN and GLD models have been extended to take into account material thermal heating due to plastic dissipation. These two constitutive laws have been implemented into the commercial finite element code Abaqus/Explicit in order to simulate the necking of a round bar and the failure of a sheet deep drawing. The results showed the importance of incorporating the shape effects of the cavities for a correct description of the material failure.
\end{abstract}

\section{Introduction}

The ductile fracture of the materials is associated with the degradation of the mechanical properties under the effect of three physical mechanisms: nucleation of the cavities, their growth under appropriate loading, and the coalescence of the neighbouring voids at an advanced deformation stage. Several nucleation criteria have been proposed in the literature. Some of these criterions have been formulated by exploiting the energetic criterion of germination, initially enunciated by Gurland and Plateau $[1,2]$. Needleman and Rice proposed a nucleation criterion depending on the inclusions rate using a phenomenological approach [3]. Other nucleation criteria have experimental origins [4]. The modelling of void growth has undergone several important contributions. Gurson [5] proposed a kinematically based limit analysis approach of a hollow sphere with an isotropic rigid-plastic matrix and developed a macroscopic yield criterion depending on the porosity. Tvergaard and Needleman extend this model to incorporate cavities interaction and to describe cavity coalescence in a phenomenological way $[6,7]$. The new model is known as GTN model. Recently Nahshon and Hutchinson [8] proposed an interesting modification of the porosity evolution law to account material damage under conditions of low or vanishing triaxiality. With the same aim Bai and Wierzbick [9], Xue [10], Xue and Wierzbicki [11], Danas and P. Ponte Castañeda [12], and Zhou et al. [13] studied the dependency of ductile fracture on the Lode angle in metals by incorporating shear mechanisms effects in the porosity evolution law. Although these new expressions do not agree with the classical porosity law derived from homogenization and having a physical interpretation, these extensions offer good results and improve them substantially at low triaxialities; see, for instance, [14-18]. All these models have been proposed by considering the matrix behaviour governed by von Mises-like model. Benzerga and Besson [19-21] carried out a series of unit cell calculations for initially spherical voids embedded now in an orthotropic matrix. Cazacu et al. [22] assumed the matrix obeying Tresca's criterion when performing limitanalysis of the hollow sphere. Gologanu et al. [23, 24] extend the Gurson analysis to introduce cavity shape effects in the socalled GLD model. They proposed a model incorporating the porosity and a new internal variable: the shape parameter. The model has been assessed by Pardoen and Hutchinson [25] in comparison to unit cell prediction with different spheroidal cavities in the case of a hardenable matrix. Ould Ouali 


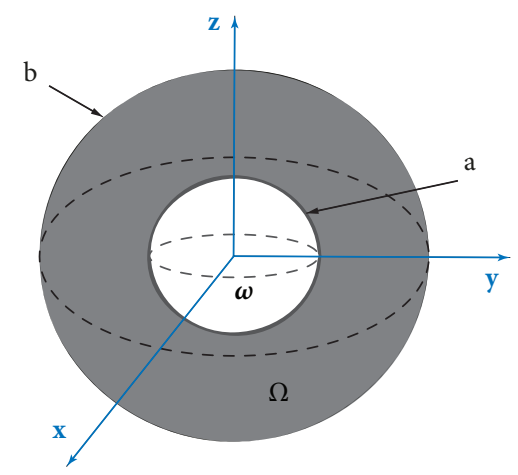

FIGURE 1: Geometry of the RVE corresponding to the Gurson model.

and Aberkane [26-28] studied namely the microstructure evolution during rolling of an A1050 aluminium sheet and introduced thermomechanical coupling in the GLD model. Madou and Leblond's [29, 30] extended the GLD model to general ellipsoidal voids having three distinct axes.

The numerical simulation of the mechanical behaviour of metals undergoing forming operations has been a great contribution to the optimization of manufacturing processes. Indeed, the study of the material failure during such operations must prevent the arising of the internal or surface cracks as well as their propagations before causing the total rupture of the structure. Given the high number of parameters controlling the deformation mechanisms under such solicitations the recourse to modelling is necessary. In the present work, the modelling includes the effects of the microstructure evolution in the material on the overall response of the part being formed. However, it is generally observed in material forming processes low triaxiality which can be an obstacle to use the classical physically based models. The purpose of the study is to assess the relevance of taking into account the evolution of cavities shape parameter as proposed in the GLD model to correctly predict the ductile failure of metals. Benzerga et al. [32] showed that the consideration of cavity shape change is currently the most appropriate physical description for modelling ductile fracture at low triaxiality. Two applications have been chosen: necking of a round bar under tensile loading and the deep drawing of an aluminium sheet. Comparison of the numerical and experimental results shows that neglecting cavity shape change leads to poor numerical predictions.

\section{Mathematical Modelling}

\subsection{Voids Growth}

2.1.1. Gurson and Tvergaard (GT) Model. Gurson [5] developed a mechanical model for porous materials by limit analysis of a spherical Representative Volume (RVE) containing a spherical confocal void (Figure 1). Tvergaard [7] proposed an extension of the Gurson plasticity criterion by introducing three coefficients $q_{1}, q_{2}$, and $q_{3}=q_{1}^{2}$ to account for the interaction between cavities. It also replaced the yield stress $\sigma_{0}$ with the flow stress $\bar{\sigma}$ in order to incorporate the isotropic work hardening of the matrix. The plastic potential is in the following form:

$$
\Phi=\frac{\sum_{e q}^{2}}{\bar{\sigma}^{2}}+2 q_{1} f \cosh \left(\frac{3}{2} q_{2} \frac{\sum_{m}}{\bar{\sigma}}\right)-1-\left(q_{1} f\right)^{2}=0
$$

where $\sum_{e q}$ is the macroscopic von Mises equivalent stress, $\sum_{m}$ the macroscopic mean stress, and $f$ the porosity of the material. The matrix hardening was introduced using the equivalence between the macroscopic and the microscopic plastic dissipation as proposed by Gurson [5]:

$$
(1-f) \dot{\bar{\sigma} \dot{\varepsilon}}^{p}=\sum: \dot{E}^{p}
$$

where $\dot{E}^{p}$ is the macroscopic plastic strain rate tensor and $\dot{\bar{\varepsilon}}^{p}$ the cumulated plastic strain rate of the matrix.

The evolution law of the porosity can be approximated using the condition of plastic incompressibility of the matrix. In the case where nucleation of new voids is not taken into account, this is given by

$$
\dot{f}=3(1-f) \dot{E}_{m}^{p}
$$

2.1.2. The GLD Model. Gologanu et al. [23, 24] extended the Gurson's analysis to a prolate or oblate ellipsoidal RVE containing a confocal cavity and subjected to some asymmetric loadings (Figure 2). The authors assumed that the voids can change their shape under the effect of loading. Such modelling requires the introduction of a new internal variable: the shape parameter $S=\ln W=\ln (a / b)$.

The expression of the plastic potential is given by the following $[21,25,26]$ :

$$
\begin{aligned}
\Phi= & \frac{C}{\bar{\sigma}^{2}}\left\|\sum^{\prime}+\eta \sum_{h} X\right\|^{2} \\
& +2 q(g+1)(g+f) \cosh \left(\frac{\kappa \sum_{h}}{\bar{\sigma}}\right)-(g+1)^{2} \\
& -q^{2}(g+f)^{2}=0
\end{aligned}
$$

where $\|$.$\| is the von Mises norm and \mathbf{X}$ a second-order tensor defined by $\mathrm{X}=(1 / 3)\left(-e_{x} \otimes e_{x}-e_{y} \otimes e_{y}+2 e_{z} \otimes e_{z}\right) . \sum_{h}$ is the hydrostatic stress weighted by coefficients depending on the cavity geometry. It governs the voids growth [19]. $\kappa$ and $g$ are the coefficients of the GLD related to porosity and shape parameter. The coefficient $\mathrm{q}$ is the equivalent of the Tvergaard coefficient in the GT model [33]:

$$
q=\tan ^{-1}[4(2.5-T)]\left|\frac{b^{-1}}{\pi}\right|+\frac{b+1}{2}
$$

where $b=1+(0.655-1.75 n-0.533 \sqrt[4]{f})\left(1 / 2+\tan ^{-1}[2(1-\right.$ $\left.S)] / \pi-0.0288 e^{-1.08(0.2+S)}\right)$.

The evolution law of the shape parameter $S$ is obtained by making some analysis and calibrations with cells calculations $[23,24]$ :

$$
\dot{S}=\frac{3}{2} h_{1}\left(\dot{E}_{z z}^{p}\right)^{\prime}+3 h_{2} \dot{E}_{m}^{p}
$$

where $h_{1}$ and $h_{2}$ are two parameters depending on $f, S$, and triaxiality $\mathrm{T}=\sum_{m} / \sum_{e q}$. 


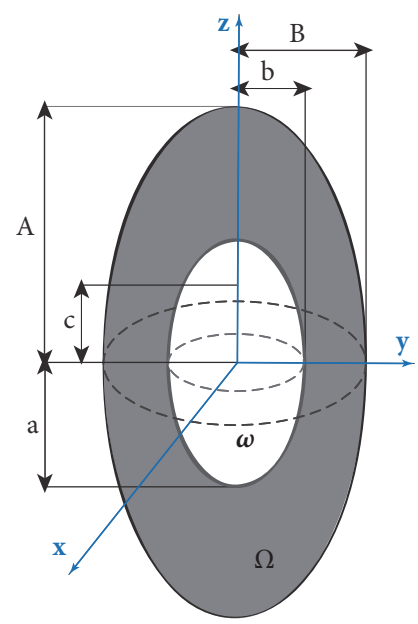

(a)

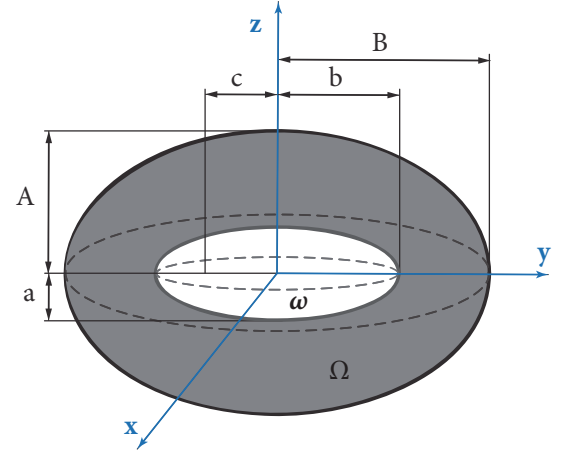

(b)

Figure 2: Geometry of the RVE corresponding to the GLD model. (a) Prolate cavity. (b) Oblate cavity.

2.2. Coalescence Stage. Prior to coalescence, the GT or GLD model is used to describe the material behaviour $[3,31]$. These models cannot capture any realistic void volume fraction during the final coalescence stage. So, the description of the material capacity loss needs to use a coalescence model. In this study, the function $f^{*}$ proposed by Tvergaard and Needleman is adopted [7]. $f^{*}$ allow the reproduction of the rapid void volumes growth at the material coalescence beginning from some critical value $f_{c}$ of $f$ :

$$
f^{*}= \begin{cases}f & \text { if } f \leq f_{c}, \\ f_{c}+\delta\left(f-f_{c}\right) & \text { otherwise }\end{cases}
$$

where $\delta=\left(f_{u}-f_{c}\right) /\left(f_{F}-f_{c}\right)$ describe the rapid increase of the porosity at the coalescence. $f_{c}$ and $f_{F}$ are the porosities at the onset of coalescence and at final failure, respectively.

In the model, the critical porosity can be determined using the Thomason's criterion. This criterion has been formulated with the assumption that the onset of coalescence occurs when the limit plastic charge is reached at the material ligament between voids (see Figure 3). The coalescence criterion is therefore written in the case of the GTN model, as follows:

$$
\begin{aligned}
& {\left[0.1\left(\frac{B-b}{a}\right)^{2}+1.2 \sqrt{\frac{B}{b}}\right]} \\
& \cdot\left[1-\left(\frac{3 \sqrt{\pi}}{4} f_{0}\right)\left(\frac{b}{R_{0}}\right) \exp \left(E_{z z}\right)\right]=0
\end{aligned}
$$

Equation (8) can be reformulated in accordance with the dimensionless variables $\chi=b / B=\left((3 / 2) f \lambda e^{-S}\right)^{1 / 3}$ and $\lambda=A / B=\lambda_{0} \exp \left((3 / 2) E_{e q}\right)$ used in the GLD model. We write the following $[19,28]$ :

$$
\frac{\sum_{z z}}{\bar{\sigma}}=\left(1-\chi^{2}\right)\left[0.1\left(\frac{1-\chi}{\chi W}\right)^{2}+1.2 \sqrt{\frac{1}{\chi}}\right]
$$

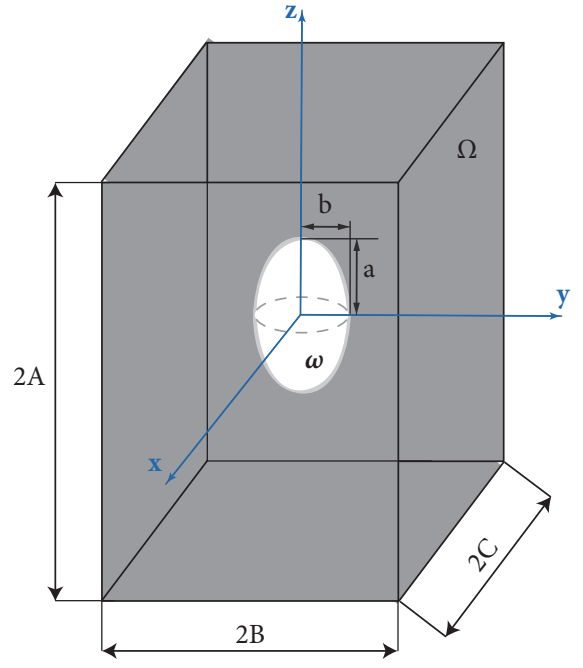

Figure 3: Geometry of Thomason's criterion.

2.3. Thermal Heating due to Plastic Dissipation. The GTN and GLD models have been extended to take into account the thermal heating due to plastic dissipation. This extension is formulated using the hypothesis of a rigid-plastic behaviour of the matrix according to Gurson assumption. The thermoplastic coupling is introduced by enforcing the dependence of the flow stress $\bar{\sigma}$, characterizing the isotropic matrix hardening, on equivalent plastic strain $\bar{\varepsilon}^{p}$ and the temperature $T[27,28]$. The following hardening law is chosen:

$$
\bar{\sigma}=\bar{\sigma}\left(\bar{\varepsilon}^{p}, T\right)=\sigma_{0}\left(\frac{\bar{\varepsilon}}{\varepsilon_{0}}\right)^{n}\left(1-\omega \frac{T}{T_{0}}\right)
$$

where $\sigma_{0}$ is the initial yield stress and $n$ hardening exponent. $\omega$ is a material parameter. 
In the absence of external heat sources, the temperature evolution law is solved with the general heat equation under adiabatic conditions (neglecting the conductivity term):

$$
\rho C \dot{T}=\xi_{f} \dot{\bar{\sigma}}^{p}
$$

where $\rho$ is the material density, $C$ is the specific heat capacity, and $\xi_{f}=\left(1-f-\rho \partial \psi / \bar{\sigma} \partial \bar{\varepsilon}^{p}\right)$ is the expression of the TaylorQuinney coefficient for porous material.

2.4. The Thermoelasticity Law. For the sake of consistency, the following thermoelastic law is introduced [27, 28]:

$$
\breve{\mathbf{\Sigma}}=\Lambda^{e}:\left(\dot{\mathbf{E}}-\dot{\mathbf{E}}^{t h}\right)+\frac{\partial \boldsymbol{\Lambda}^{e}}{\partial T}:\left(\mathbf{E}-\mathbf{E}^{t h}\right) \dot{T}
$$

where $\mathbf{E}^{\text {th }}=\alpha\left(T-T_{O}\right) \mathbf{I}$ is the strain tensor due to thermal expansion and $\breve{\Sigma}$ some objective derivative of the stress tensor. I is the second-order unit tensor.

2.5. Numerical Implementation. The GTN and GLD models have been implemented into the finite element code Abaqus/Explicit using the Vectorized User MATerial (VUMat) subroutine and used to carry out the numerical simulations. The "elastic predictor-plastic correction" algorithm proposed by Aravas [34] is adopted in the local resolution of the constitutive laws GTN and GLD. The details of the implementation of these two behaviour laws, as well as the validation in comparison with the predictions of cell calculations using an explicit technique, are given in $[26,27,33]$.

Aravas's algorithm is based on the decomposition of the stress tensor into its hydrostatic $\sigma_{m}$ and deviator parts $\sigma_{e q}$ and the plastic strain increment tensor $\Delta \varepsilon^{p}$ into its volumetric and deviator parts:

$$
\begin{gathered}
\boldsymbol{\sigma}=\sigma_{m} \mathbf{1}+\frac{2}{3} \sigma_{e q} \mathbf{n} \\
\Delta \boldsymbol{\varepsilon}^{p}=\frac{2}{3} \Delta \varepsilon_{\mathrm{p}} \mathbf{1}+\Delta \varepsilon_{q} \mathbf{n}
\end{gathered}
$$

$\Delta \varepsilon_{\mathrm{p}}$ and $\Delta \varepsilon_{q}$ are used by Aravas as primary variables. So, the system is reduced to two non-inear equations:

$$
\begin{aligned}
\Delta \varepsilon_{\mathrm{p}} \frac{\partial \Phi}{\partial \sigma_{e q}}+\Delta \varepsilon_{q} \frac{\partial \Phi}{\partial \sigma_{m}} & =0 \\
\Phi(\sigma, f, \bar{\sigma}) & =0
\end{aligned}
$$

After the resolution of (15) and (16), the hydrostatic stress $\Sigma_{m}$ and the equivalent stress $\Sigma_{e q}$ are updated:

$$
\begin{aligned}
& \Sigma_{m}=\Sigma_{m}^{e}+K \Delta E_{p} \\
& \Sigma_{e q}=\Sigma_{e q}^{e}-3 G \Delta E_{q}
\end{aligned}
$$

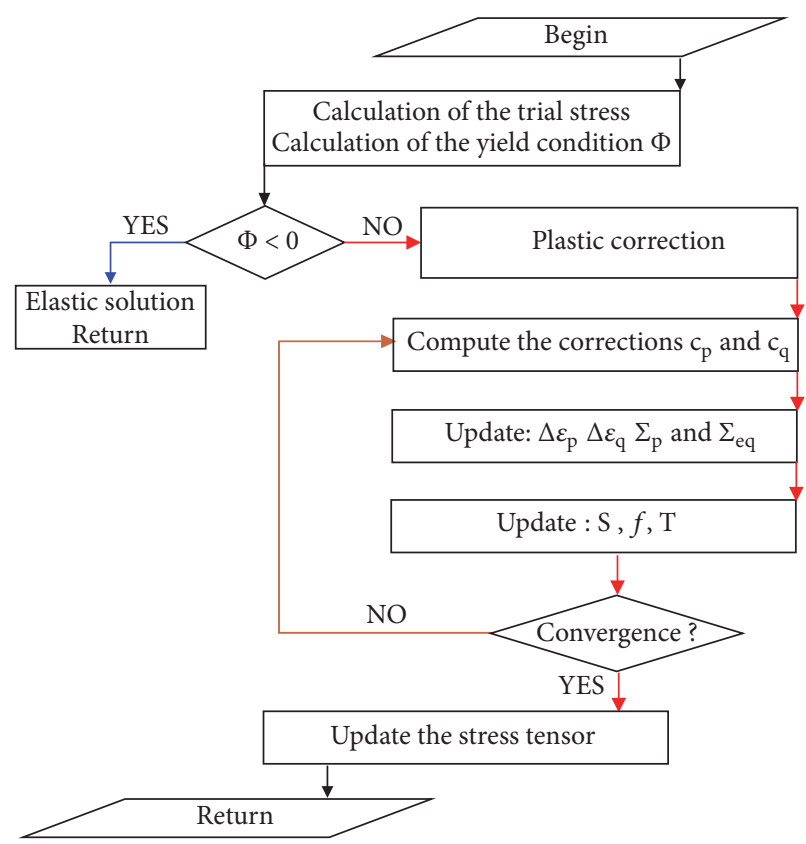

FIgURE 4: Flowchart of the implementation of the GLD model.

Then the state variables are actualized according to the following:

$$
\begin{aligned}
\Delta f & =(1-f) \Delta E_{p}+\mathscr{A} \Delta E_{m}^{p l} \\
\Delta S & =\frac{3}{2} h_{1}\left(\frac{1}{3} \Delta E_{p}+n \Delta E_{q}\right)+h_{2} \Delta E_{p} \\
\Delta T & =\frac{\xi}{\rho C} \frac{-\Sigma_{m} \Delta E_{p}+\Sigma_{e q} \Delta E_{q}}{(1-f)} \\
\Delta \bar{\sigma} & =\left(\frac{\hbar_{\mathscr{C}}}{\bar{\sigma}}+\hbar_{T} \frac{\xi}{\rho c_{v}}\right) \frac{-\Sigma_{m} \Delta E_{p}+\Sigma_{e q} \Delta E_{q}}{(1-f)}
\end{aligned}
$$

where $\hbar_{\mathscr{C}}=\partial \bar{\sigma} / \partial \bar{\varepsilon}^{p}$ and $\hbar_{\mathscr{T}}=\partial \bar{\sigma} / \partial T$ are, respectively, the plastic and thermal modulus.

Details of the implementation procedure are done in the flowchart of Figure 4 and can be found in [19, 20, 24].

It is important to notice the GLD growth model implementation requires specific treatment due to the "limits" shapes that the cavities can take during the loading process: penny-shaped crack $(f \longrightarrow 0, S \longrightarrow-\infty)$, "sandwich" structure $(f \equiv$ finite, $\mathrm{S} \longrightarrow-\infty)$, spherical $(f \equiv$ finite, $\mathrm{S}=0)$ or cylindrical $(f \equiv$ finite, $S \longrightarrow+\infty)$ shapes, or a needle $(f \longrightarrow$ $0, S \longrightarrow+\infty)$. Specific numerical treatment of the particular expressions of the plastic potential $\Phi$ according to each case should be carried out.

\section{Results and Discussion}

In this section, we discuss the results obtained with the GTN and GLD models extended to take account the thermal heating due to plastic dissipation and to predict the onset of coalescence using Thomason's criterion. In order to highlight the effect of cavity shape change, we make use of the same 
TABLE 1: Material parameters used with the GTN and GLD models.

\begin{tabular}{lcccccccc}
\hline & $S_{0}$ & $f_{0}$ & $f_{c}(\%)$ & $f_{F}(\%)$ & $f_{N}(\%)$ & $\varepsilon_{N}$ & $S_{N}$ & $q$ parameters \\
\hline GTN & - & 0 & 15 & 25 & 4 & 0.3 & 0.1 & $q_{0}=1.6$ \\
GLD & 0 & 0 & 15 & 25 & 4 & 0.3 & 0.1 & $q_{1}=1.5, q_{2}=1.1$ \\
\hline
\end{tabular}

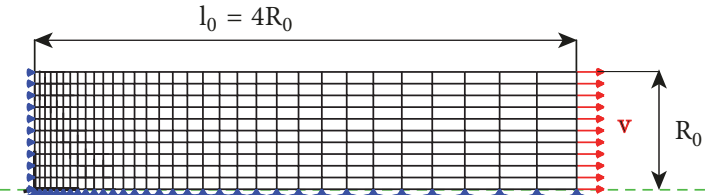

(a)

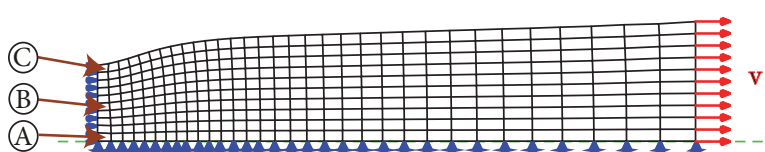

(b)

FIgure 5: Cylindrical bar. (a) Initial mesh. (b) Deformed mesh at failure.

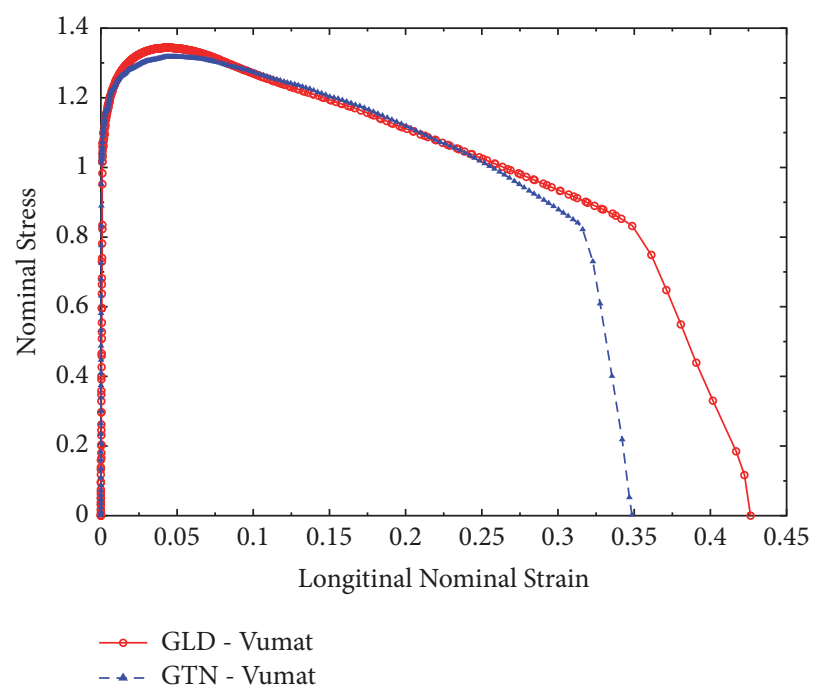

(a)

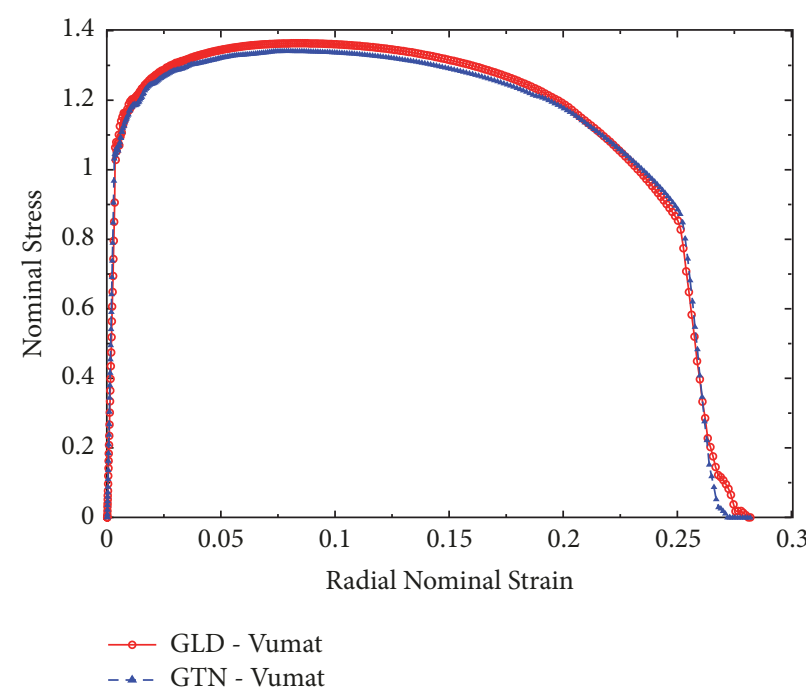

(b)

FIGURE 6: Evolution of the nominal stress in function of (a) nominal strain and (b) radius reduction.

material parameters for both the GTN and GLD models with the exception of those related to the state variable describing the cavity shape change: the cavity shape parameter is not described in the GTN model. Two applications were chosen: tensile of a round specimen and deep drawing of a sheet.

\subsection{Necking of Cylindrical Bar. The GTN and GLD models} are used to simulate the rupture of a round bar subjected to tensile loading. It has been found experimentally that the failure of the bar occurs according to the so-called "cup cone" mode $[7,35]$. Indeed, Thomason observed that the crack appears in the centre zone of the specimen and then spreads horizontally in the plane perpendicular to the loading axis. Near of the outer surface, the crack deviates with an angle of $45^{\circ}$ from the propagation direction forming a cup cone fracture surface.

The height/radius ratio of the cylindrical bar is taken equal to $l_{0} / R_{0}=4$. Three hundred and twenty (320) axisymmetric elements with reduced integration (CAXR) are used to mesh the quarter of the specimen. The initial and deformed meshes, as well as the loading conditions, are shown in Figure 5. The constituent material of the specimen is a standard steel with a rigidity $E / \sigma_{0}=500$ and Poisson's ratio $\nu=0.28$. The hardening exponent $n=0.1$.

The material is assumed to be initially dense $\left(f_{0}=0\right)$. We use the same coalescence and nucleation parameters as those used by Tvergaard and Needleman when studying necking of the cylindrical bar [7]. These parameters are done in Table 1.

The evolution of the nominal stress $\sigma_{N}=F / \pi R_{0}^{2} \sigma_{0}$ versus the longitudinal nominal strain $\varepsilon_{L}=u / l_{0}$ and the radial nominal strain (reduction of radius) $\varepsilon_{R}=\Delta R / R_{0}$ calculated with the two models GTN and GLD are represented in Figure 6. During the test, the nominal stress $\sigma_{N}$ increases to a maximum that corresponds to the onset of necking, then decreases [7]. This diminution of the nominal stress is accentuated at the end of the test because of the coalescence of the cavities until the final rupture. A difference appears in nominal stress levels reached by the GTN and the GLD models. This difference can be explained by the effect of the cavity shape change on the resistance of the materials 


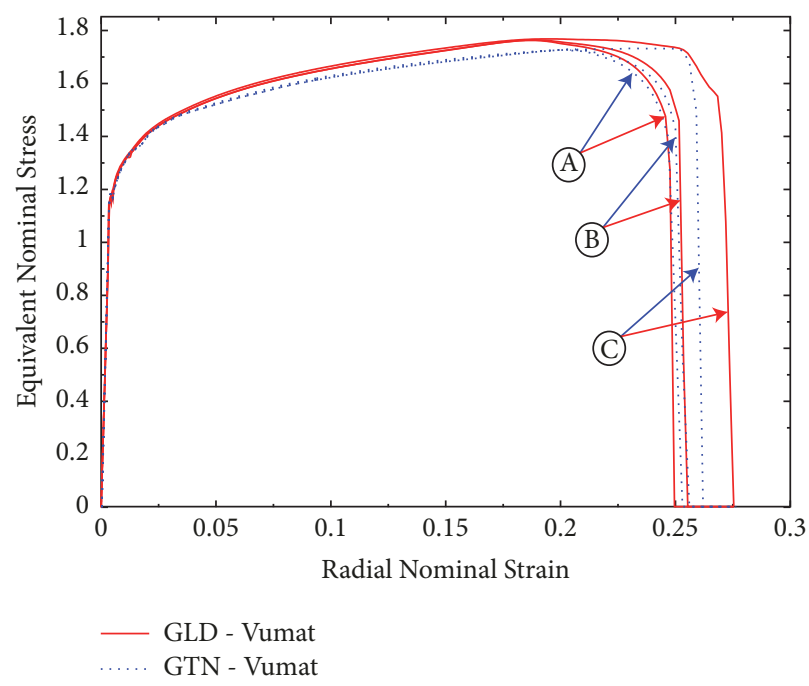

(a)

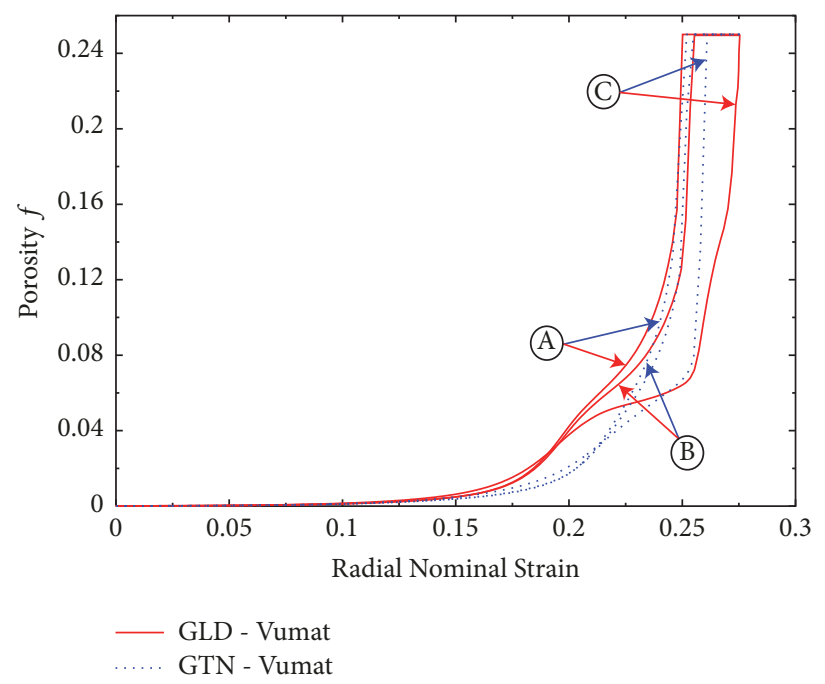

(b)

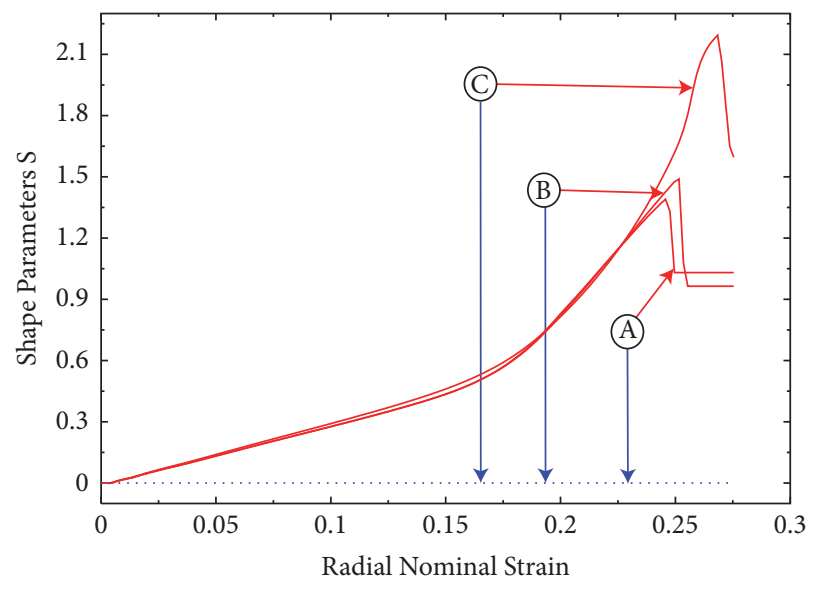

(c)

Figure 7: Evolution of the stress state and the microstructure at the neck. (a) Equivalent nominal stress $\sum_{\text {eq }} / \sigma_{0}$. (b) The porosity $f$. (c) The shape parameter $S$.

observed, namely, in cell calculations [19, 24-26, 33]. These authors have related these results to the morphological anisotropy generated by the cavities shape that the GLD model allows to take into account.

In the case of a simulation with the GLD model, the maximum force is equal to $F_{\max }^{G L D}=1.344 \pi R_{0}^{2} \sigma_{0}$ whereas that predicted by the GTN model is $F_{\max }^{G T N}=1.311 \pi R_{0}^{2} \sigma_{0}$. The two maxima are obtained for the same radial nominal strain $\varepsilon_{R}^{\text {neck }}=0.0847$ and for close longitudinal strains of about $\varepsilon_{L}^{\text {neck }}=0.04$. These main results are reported in Table 2 .

$\varepsilon_{L}^{\text {fail }}$ and $\varepsilon_{R}^{\text {fail }}$ are, respectively, the longitudinal and the radial nominal strain of the bar at failure. A qualitative explanation of this difference was given by Aravas and Ponte Castañeda concerning this test using the model proposed by Aravas and Ponte Castañeda [36]. In the case of the GLD model, the justification can be done regarding the evolution of the microstructure (the porosity $f$ and the shape
TABLE 2: Representative values of the stress-strain curves corresponding to a cylindrical bar under tensile load.

\begin{tabular}{lccccc}
\hline & $\sigma_{N}$ & $\varepsilon_{R}^{\text {neck }}$ & $\varepsilon_{L}^{\text {neck }}$ & $\varepsilon_{R}^{\text {fail }}$ & $\varepsilon_{L}^{\text {fail }}$ \\
\hline GTN & 1.311 & 0.0847 & 0.0448 & 0.261 & 0.348 \\
GLD & 1.344 & 0.0847 & 0.0433 & 0.275 & 0.426 \\
\hline
\end{tabular}

parameter $S$ ) at the neck. Figure 7 represents the evolution of the nominal von Mises stress $\sum_{e q} / \sigma_{0}$, the porosity $f$, and the shape parameter $S$ versus the nominal radial strain in three elements (A), (B), and (C) of the bar located at the neck (see Figure 7). It is well-known that the effective nominal stress depends on the net area of the section (without voids) perpendicular to the load at the neck that supports the load in the case of tensile loading. This area decreases when the total area of the section of the bar decreases (necking) and/or 
when the porosity in the plane carrying the load increases. As previously mentioned, the section of the bar for which the difference between the maximum force predicted by the GLD and GTN models is observed for the same radius reduction $\Delta \mathrm{R} / \mathrm{R}_{0}=0.0847$. Consequently, the difference in the intensity of the loading force can only be justified by the second point: the surface occupied by the voids at the neck of the bar.

We will study this point in more detail by considering the material microstructure evolution of the bar (Figures 7(b) and $7(\mathrm{c}))$.

(i) Figure 7(b) shows that the porosity predicted by the GTN and GLD models are the same for the three elements located at the neck of the bar. These curves coincide almost at least until the nominal radial strain $\varepsilon_{R}=0.0847$ which corresponds to the onset of necking.

(ii) In Figure 7(c), we can see that the voids tend to lengthen in the direction of loading, in the case of the GTN model, since the shape parameter S increases. For the GTN model, the cavities keep the spherical shape. It follows that, for the same porosity, the area occupied by the voids in the section at the neck is smaller in the case of the GLD model: the cavities shape have changed from spherical (initial shape $S=$ $0)$ to elongated voids $(S>0)$.

So, for the same volume fraction of voids, the surface of the voids in the section of the neck supporting the load is smaller in the case of the GLD model compared to the GTN model. Indeed, during the simulation with the GTN model the increase of the porosity is done by the increase of the diameter of the void. So, the effective surface is smaller than that predicted by the GLD model. Knowing that the effective stress is directly related to the applied force, the difference between the effective surfaces explains the difference between the maximum forces achieved.

3.2. Deep Drawing of a Sheet. The deep drawing of a thin sheet of aluminium alloy is studied. We are interested in the Swift deep drawing test which is applied to an aluminium sheet having a thickness of $1 \mathrm{~mm}$, until failure [31]. The dimensions of the sheet and the tools are shown in Figure 8. Due to geometry and applied load symmetries, the test is modelled under axisymmetric conditions. The mesh of the sheet is composed of 640 axisymmetric elements with reduced integration CAXR with 04 layers of elements on the thickness. The friction between the sheet and the tools has been determined experimentally by the CETIM/Senlis (France) and is equal to $\mu_{f}=0.17$ [31]. The clamping force applied by the blanking tool is $F_{s}=5 \mathrm{kN}$.

The material properties of the aluminium alloy sheet are Young's modulus $E=70 \mathrm{GPa}$, Poisson's ratio $v=0.33$, density $\rho=2700 \mathrm{Kg} / \mathrm{m}^{3}$, and yield strength $\sigma_{0}=114 \mathrm{MPa}$. The hardening exponent is $n=0.146$. In the absence of experimental data on the microstructure of the aluminum alloy, we have adopted the initial porosity: $f_{0}=0.1 \%$. This level of initial porosity is generally observed in such material. For choosing the initial value of the shape parameter, a

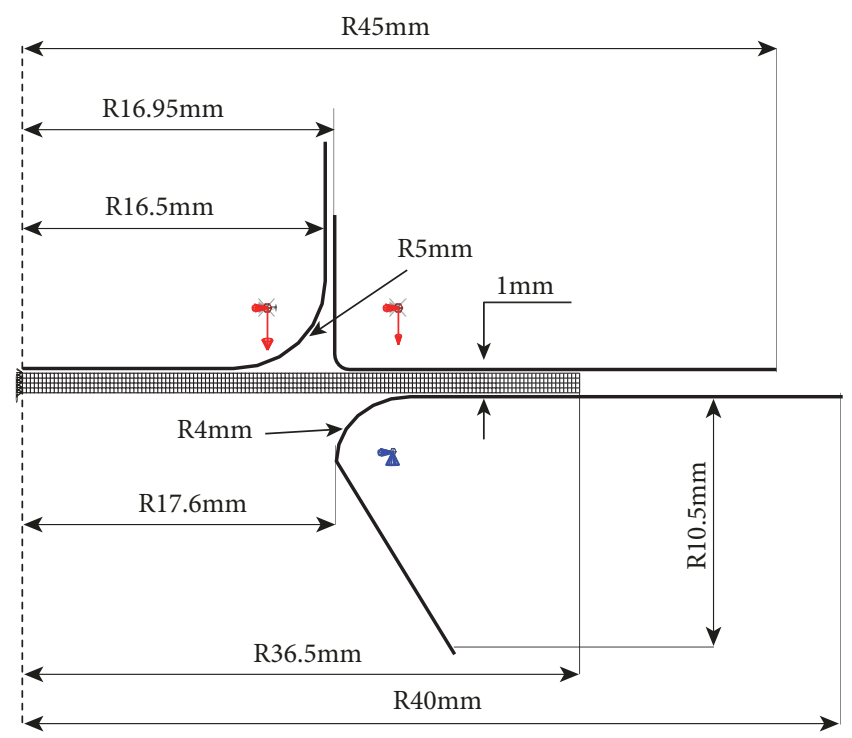

FIGURE 8: Schematization of the deep drawing test: initial mesh and boundary conditions.

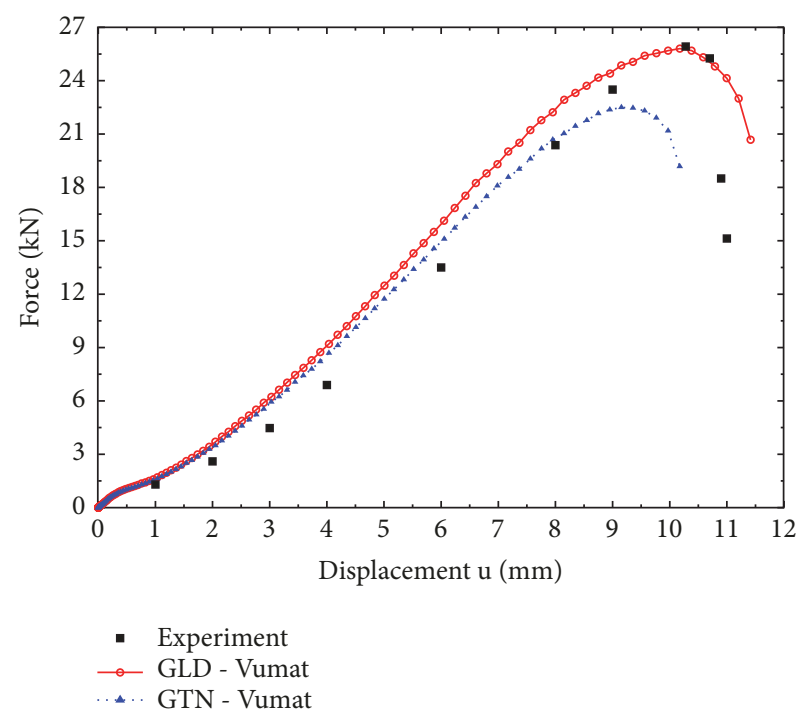

FIGURE 9: Evolution of the stamping force versus tool displacement [31].

parametric study has been carried out using initial oblate, spherical, and prolate cavities. It is found that a value of $S_{0}=$ 1.38 gives good results in this case.

Figure 9 shows a comparison between the experimental results obtained by the CETIM/Senlis with the numerical predictions of the GTN and GLD models. The curves describe the evolution of the deep drawing force as a function of the punch displacement. Good agreement is found between experimental and numerical results in the case of the GLD model. However, a difference appears during the simulation with the GTN model.

Table 3 resumes the main values of the forcedisplacement curve during deep drawing of the aluminium sheet: maximal force and corresponding displacement. In 


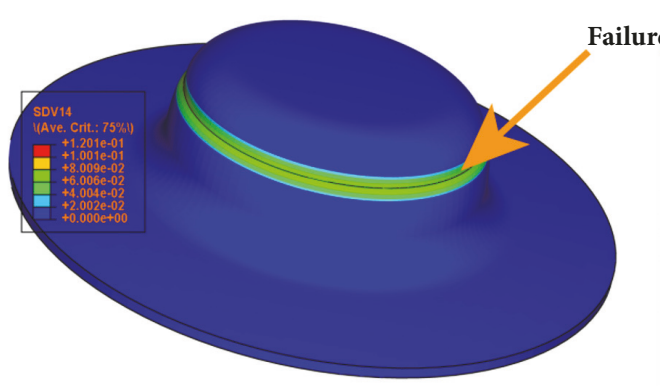

(a)

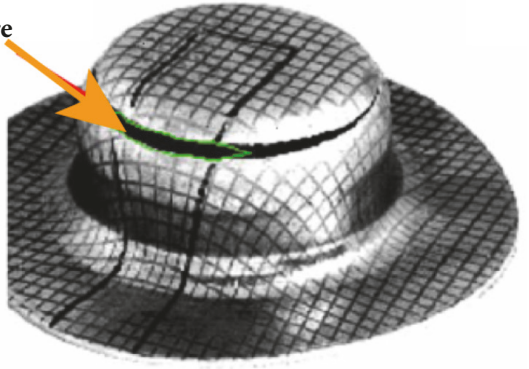

(b)

FIgURE 10: Distribution of the porosity in the sheet at failure and experiment [31].

TABLE 3: Representative points of the force-displacement curve during deep drawing of the sheet.

\begin{tabular}{lccc}
\hline & Experiment & GTN & GLD \\
\hline Maximal force $(\mathrm{kN})$ & 25.92 & 22.31 & 25.66 \\
Error $(\%)$ & - & 13.93 & 1.00 \\
\hline Displacement $(\mathrm{mm})$ & 10.28 & 9.19 & 10.19 \\
Error $(\%)$ & - & 10.60 & 0.87 \\
\hline
\end{tabular}

the estimation of the error, the experimental results are taken as reference. The error made during the simulation with the GTN model (13.93\% and $10.6 \%)$ is more important than that made using the GLD model (1\% and $0.87 \%$ ). An explanation of these differences can be found regarding the orthotropic behaviour of the sheet experimentally identified by the CETIM/Senlis [31]. In the GTN model, the matrix behaviour is assumed to be isotropic. In the GLD model, a morphological anisotropy is introduced by the change of the ellipsoidal cavities shape.

Figure 10 shows comparison of the stamp shape obtained experimentally and that described numerically with the GLD model. We observe that the two geometries are practically identical and that the rupture appears in the same region of the sheet.

\section{Conclusions}

Two physically based constitutive laws, the GTN and the GLD models, have been implemented in the ABAQUS calculation via the VUMAT subroutine. Both models have been extended to account for thermal heating due to plastic dissipation under adiabatic conditions. The theoretical and numerical aspects of these formulations are detailed. These models have been used for the simulation of a round bar under tensile loading and deep drawing of aluminium sheet. From the comparison of the predictions of these models, we can state that incorporating cavity shape changes is an important step in the correct modelling of the ductile failure of metals

As a perspective for this work, we plan to carry out experimental tests and unit cell calculations in order to verify the pertinence of the shape parameter evolution law under different stress states. Especially in the case of thermomechanical loading, another interesting extension is to introduce the orthotropic material behaviour existing, namely, in thin structures. Finally, the incorporation of shear mechanisms will also significantly improve the numerical predictions of ductile fracture arising in some particular engineering problems.

\section{Data Availability}

Numerical data used to support the findings of this study are actually restricted by a research convention. Requests for data, after publication of this article, can be addressed to the corresponding author for consideration in the context of the convention.

\section{Conflicts of Interest}

The author declares that there are no conflicts of interest regarding the publication of this paper.

\section{Acknowledgments}

The author acknowledges thankfully the General Council of the Ardennes (France) for the financial support during the preparation of part of this work.

\section{References}

[1] A. S. Argon, J. Im, and R. Safoglu, "Cavity formation from inclusions in ductile fracture," Metallurgical Transactions A, vol. 6, no. 3, pp. 825-837, 1975.

[2] J. Gurland and J. Plateau, "Mechanism of ductile rupture of metals rupture of metals containing inclusions," Journal of the ASM, vol. 56, pp. 442-455, 1963.

[3] A. Needleman and J. R. Rice, "Limit to ductility set by plastic flow localization," in Mechanics of Sheet Metal Forming, D. P. Koistinie and N. M. Wang, Eds., pp. 237-267, Plenum Press, New York, NY, USA, 1978.

[4] F. M. Beremin, "Cavity formation from inclusions in ductile fracture of A508 steel," Metallurgical Transactions A, vol. 12, no. 5, pp. 723-731, 1981.

[5] A. L. Gurson, "Continuum theory of ductile rupture by void nucleation and growth : part I-yield criteria and flow rules for porous ductile media," Journal of Engineering Materials and Technology, vol. 99, no. 1, pp. 2-15, 1977.

[6] J. W. Hancock and R. D. Thomson, "Strain and stress concentrations in ductile fracture by void nucleation growth and 
coalescence," Materials Science and Technology, vol. 1, no. 9, pp. 684-690, 1985.

[7] V. Tvergaard and A. Needleman, "Analysis of the cup-cone fracture in a round tensile bar," Acta Metallurgica et Materialia, vol. 32, no. 1, pp. 157-169, 1984.

[8] K. Nahshon and J. W. Hutchinson, "Modification of the Gurson Model for shear failure," European Journal of Mechanics A/Solids, vol. 27, no. 1, pp. 1-17, 2008.

[9] Y. Bai and T. Wierzbicki, "Application of extended MohrCoulomb criterion to ductile fracture," International Journal of Fracture, vol. 161, no. 1, pp. 1-20, 2010.

[10] L. Xue, "Constitutive modeling of void shearing effect in ductile fracture of porous materials," Engineering Fracture Mechanics, vol. 75, no. 11, pp. 3343-3366, 2008.

[11] L. Xue and T. Wierzbicki, "Ductile fracture initiation and propagation modeling using damage plasticity theory," Engineering Fracture Mechanics, vol. 75, no. 11, pp. 3276-3293, 2008.

[12] K. Danas and P. Ponte Castañeda, "Influence of the Lode parameter and the stress triaxiality on the failure of elastoplastic porous materials," International Journal of Solids and Structures, vol. 49, no. 11-12, pp. 1325-1342, 2012.

[13] J. Zhou, X. Gao, J. C. Sobotka, B. A. Webler, and B. V. Cockeram, "On the extension of the Gurson-type porous plasticity models for prediction of ductile fracture under shear-dominated conditions," International Journal of Solids and Structures, vol. 51, no. 18, pp. 3273-3291, 2014.

[14] M. Achouri, G. Germain, P. Dal Santo, and D. Saidane, "Experimental characterization and numerical modeling of micromechanical damage under different stress states," Materials and Corrosion, vol. 50, pp. 207-222, 2013.

[15] M. Achouri, G. Germain, P. Dal Santo, and D. Saidane, "Numerical integration of an advanced Gurson model for shear loading: Application to the blanking process," Computational Materials Science, vol. 72, pp. 62-67, 2013.

[16] K. L. Nielsen, J. Dahl, and V. Tvergaard, "Collapse and coalescence of spherical voids subject to intense shearing: Studied in full 3D," International Journal of Fracture, vol. 177, no. 2, pp. 97108, 2012.

[17] V. Tvergaard, "Effect of stress-state and spacing on voids in a shear-field," International Journal of Solids and Structures, vol. 49, no. 22, pp. 3047-3054, 2012.

[18] Z. Xue, J. Faleskog, and J. W. Hutchinson, "Tension-torsion fracture experiments - Part II: Simulations with the extended Gurson model and a ductile fracture criterion based on plastic strain," International Journal of Solids and Structures, vol. 50, no. 25-26, pp. 4258-4269, 2013.

[19] A. A. Benzerga, Rupture ductile des tôles anisotropes : simulation de la propagation longitudinale dans un tube pressurisé [Ph.D. thesis], École Nationale Supérieure des Mines de paris, paris, France, 2000.

[20] A. A. Benzerga, "Micromechanics of coalescence in ductile fracture," Journal of the Mechanics and Physics of Solids, vol. 50, no. 6, pp. 1331-1362, 2002.

[21] A. A. Benzerga, J. Besson, and A. Pineau, "Anisotropic ductile fracture: Part II: Theory," Acta Materialia, vol. 52, no. 15, pp. 4639-4650, 2004.

[22] O. Cazacu, B. Revil-Baudard, N. Chandola, and D. Kondo, "New analytical criterion for porous solids with Tresca matrix under axisymmetric loadings," International Journal of Solids and Structures, vol. 51, no. 3-4, pp. 861-874, 2014.
[23] P. Suquet, Recent Extensions of Gurson's Model for Porous Ductile Metals, CISM Lectures Series, Springer, New-York, NY, USA, 1997.

[24] Mihai. Gologanu, Etude de quelques problèmes de rupture ductile des métaux [Ph.D. thesis], Université de Paris, Paris, France, 1997.

[25] T. Pardoen and J. W. Hutchinson, "Extended model for void growth and coalescence," Journal of the Mechanics and Physics of Solids, vol. 48, no. 12, pp. 2467-2512, 2000.

[26] M. Ould Ouali, Approche micromécanique de la rupture ductile dans les procédés de mise en forme des matériaux. Prise en compte de l'effet de forme des cavités [Ph.D. thesis], Université de Reims Champagne-Ardenne, Reims, France, 2007.

[27] M. Ould Ouali and M. Aberkane, "Multiscales modeling of microstructure evolution during asymmetric cold rolling process," International Journal of Material Forming, vol. 1, no. 1, pp. 89-92, 2008.

[28] M. Ould Ouali and M. Aberkane, "Micromechanical modeling of the rolling of a A1050P aluminum sheet," International Journal of Material Forming, vol. 2, no. 1, pp. 25-36, 2009.

[29] K. Madou and J.-B. Leblond, "A Gurson-type criterion for porous ductile solids containing arbitrary ellipsoidal voids II: Determination of yield criterion parameters," Journal of the Mechanics and Physics of Solids, vol. 60, no. 5, pp. 1037-1058, 2012.

[30] K. Madou, J.-B. Leblond, and L. Morin, "Numerical studies of porous ductile materials containing arbitrary ellipsoidal voids - II: Evolution of the length and orientation of the void axes," European Journal of Mechanics - A/Solids, vol. 42, pp. 490-507, 2013.

[31] M. Khelifa, M. Oudjene, and A. Khennane, "Fracture in sheet metal forming: Effect of ductile damage evolution," Computers \& Structures, vol. 85, no. 3-4, pp. 205-212, 2007.

[32] A. A. Benzerga, J.-B. Leblond, A. Needleman, and V. Tvergaard, "Ductile failure modeling," International Journal of Fracture, vol. 201, no. 1, pp. 29-80, 2016.

[33] L. Siad, M. Ould Ouali, and A. Benabbes, "Comparison of explicit and implicit finite element simulations of void growth and coalescence in porous ductile materials," Materials and Corrosion, vol. 29, no. 2, pp. 319-329, 2008.

[34] N. Aravas, "On the numerical integration of a class of pressure-dependent plasticity models," International Journal for Numerical Methods in Engineering, vol. 24, no. 7, pp. 1395-1416, 1987.

[35] P. F. Thomason, Ductile fracture of Metals, Pergamon Press, Oxford, Uk, 1990.

[36] N. Aravas and P. Ponte Castaneda, "Numerical methods for porous metals with deformation-induced anisotropy," Computer Methods Applied Mechanics and Engineering, vol. 193, no. 36-38, pp. 3767-3805, 2004. 


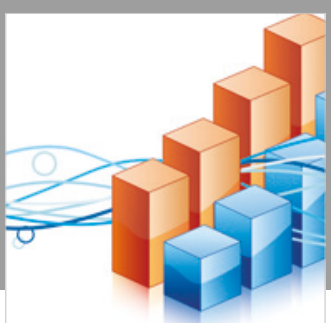

Advances in

Operations Research

\section{-n-m}
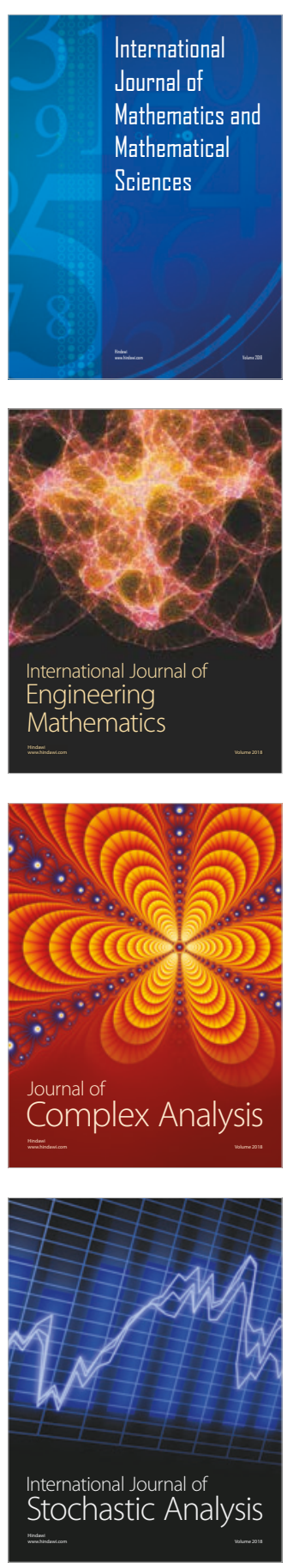
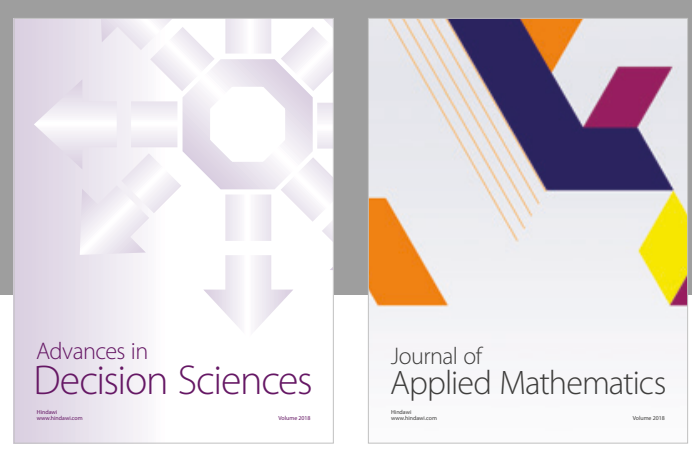

Journal of

Applied Mathematics
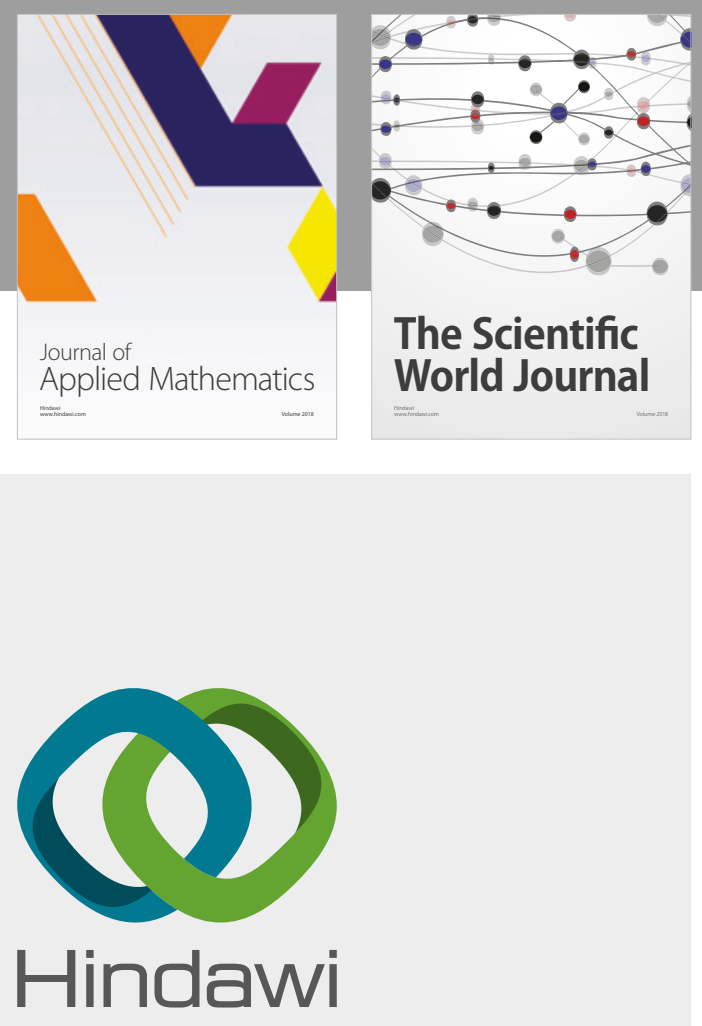

Submit your manuscripts at

www.hindawi.com

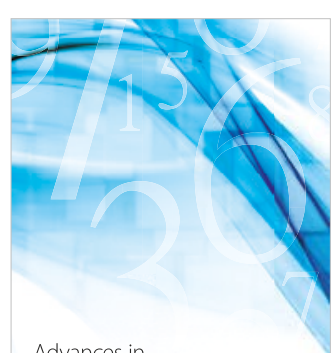

Advances in
Numerical Analysis
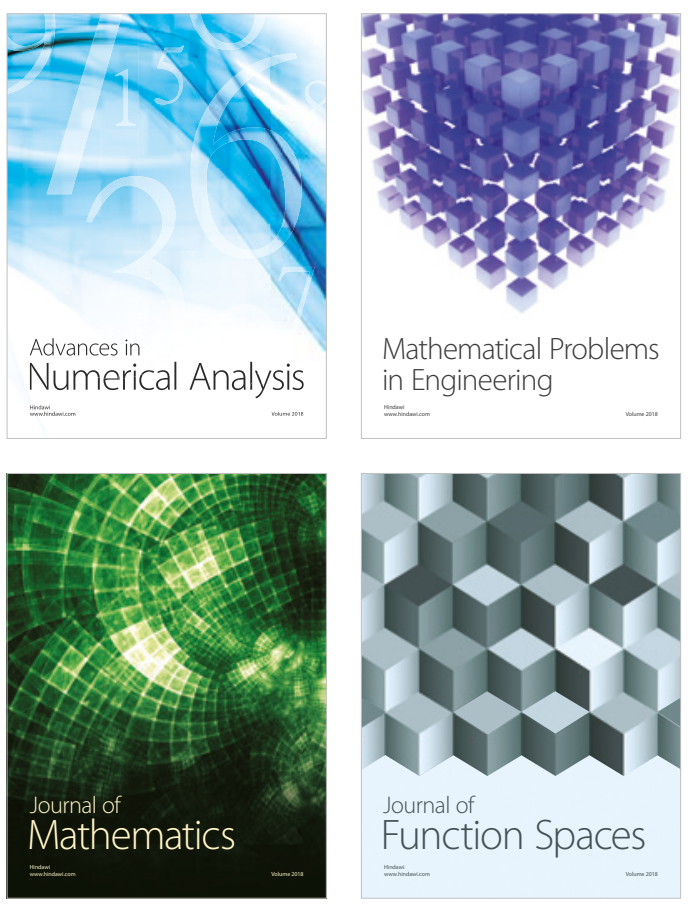

Mathematical Problems in Engineering

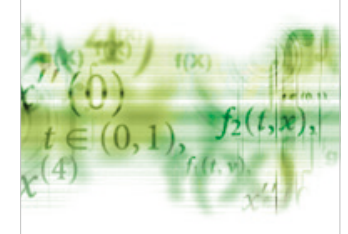

International Journal of

Differential Equations

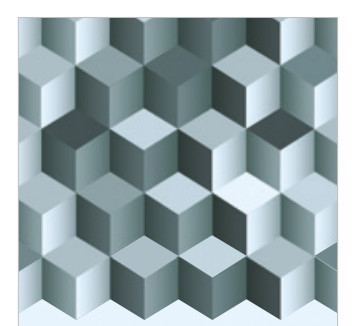

Journal of

Function Spaces

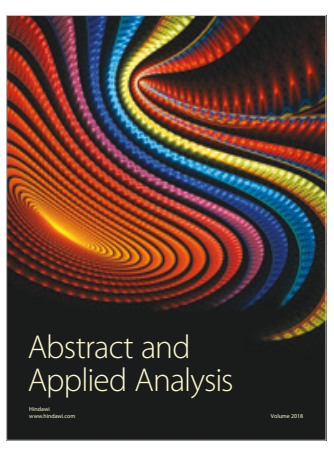

The Scientific

World Journal

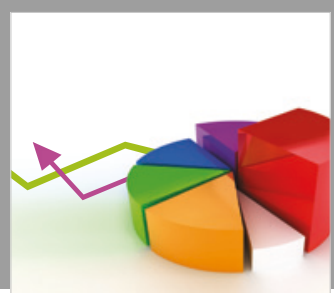

Journal of

Probability and Statistics
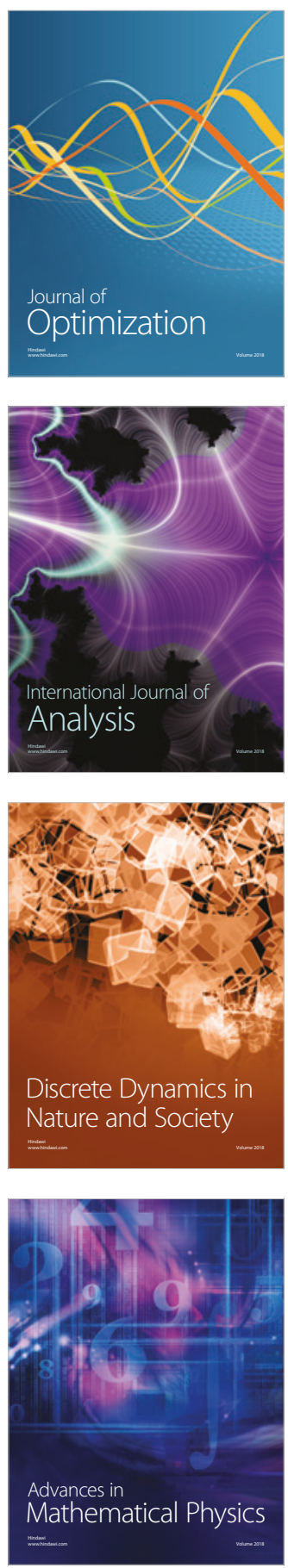